MODELING, IDENTIFICATION AND CONTROL, 1996, vOL. 17, NO. 2, 135-15I

doi:10.4173/mic.1996.26

\title{
Design of a dynamic positioning system using model-based control
}

\author{
ASGEIR J. SØRENSEN $\dagger$, SVEIN I. SAGATUN $\ddagger$ and THOR I. FOSSEN§
}

Keywords: Dynamic positioning, model-based control, hydrodynamics, sea trials, marine systems.

\begin{abstract}
A dynamic positioning (DP) system includes different control functions for automatic positioning and guidance of marine vessels by means of thruster and propeller actions. This paper describes the control functions which provide station-keeping and tracking. The DP controller is designed using model-based control, where a new modified LQG feedback controller and a model reference feedforward controller are applied. A reference model calculates appropriate reference trajectories. Since it is not desirable nor even possible to counteract the wave-frequency movement caused by first-order wave loads, the control action of the propulsion system should be produced by the low frequency part of the vessel movement caused by current, wind and second-order mean and slowly varying wave loads. A Kalman filter based state estimator and a Luenberger pbserver are used to compute the low-frequency feedback and feedforward control signals. Full-scale experiments with a supply vessel demonstrate the performance of the proposed controller.
\end{abstract}

\section{Introduction}

Dynamic positioning (DP) systems have been commercially available for marine vessels since the 1960's. The first DP systems were designed by using conventional PID controllers in cascade with low pass and/or notch filters. From the middle of the 1970's more advanced control techniques based on optimal control and Kalman filter theory were proposed by Balchen et al. (1976). This work has later been modified and extended by Balchen et al. (1980a, b), Grimble et al. (1980a, b), Fung and Grimble (1983) and Saelid et al. (1983).

In this paper a model-based control scheme which provides both station-keeping and tracking (also denoted as marked position) of ships is proposed. This algorithm is an extension of the modified LQG control algorithm proposed by Balchen (1993). A reference model is introduced in order to compute appropriate reference trajectories. Since it is not desirable to counteract the wave-frequency (WF) movement caused by first-order wave loads, the control action of the propulsion system is produced by the low-frequency (LF) part of the vessel movement caused by current, wind and second-order mean and slowly varying wave loads. A Kalman filter based state

Received 20 August 1995.

$\dagger$ ABB Industri AS, Hasleveien 50, P.O. Box 6540 Rodeløkka, N-0501 Oslo, Norway (E-mail: asgeir.sorensen@noina.abb.telemax.no).

‡ABB Industri AS, Hasleveien 50, P.O. Box 6540 Rodeløkka, N-0501 Oslo, Norway (E-mail: sis@ttsint.no).

$\S$ The Norwegian Institute of Technology, Department of Engineering Cybernetics, N-7034 Trondheim. Norway (E-mail: tif@itk.unit.no).

Reprinted from Control Engineering Practice, 1996, with kind permission from Elsevier Science Ltd, Oxford, UK. 
estimator and a Luenberger observer are used to compute the feedback and feedforward control signals.

\section{Mathematical modeling}

In order to achieve good performance of the control system it is necessary to derive a sufficient detailed mathematical model of the vessel and thruster system. The model parameters are identified by running an off-line parallel extended Kalman filter algorithm, see Fossen et al. (1995).

\subsection{Kinematics}

The different reference frames used in dynamic positioning are illustrated in Fig. 1 and described below.

- The earth-fixed reference frame is denoted as the $X_{E} Y_{E} Z_{E}$-frame.

- The vessel-parallel frame denoted as the $X_{V} Y_{V} Z_{V}$-frame is fixed in the earth-fixed frame and rotated to the desired heading angle $\psi_{0}$ and then translated to the desired $x_{0}$ and $y_{0}$ position coordinates.

- The body-fixed frame denoted as the XYZ-frame is fixed to the vessel body with the origin located at the centre of gravity

The linear velocity of the ship in the body-fixed frame relative to the earth-fixed frame is given by the transformation

$$
\dot{\boldsymbol{\eta}}=\boldsymbol{J}(\boldsymbol{\eta}) \boldsymbol{v}
$$

where the earth-fixed vessel position and heading, and the body-fixed surge, sway and yaw velocities are defined by the vectors $\boldsymbol{\eta}=[x, y, \psi]^{\mathrm{T}}$ and $\boldsymbol{v}=[u, v, r]^{\mathrm{T}}$. The rotation matrix $\boldsymbol{J}(\boldsymbol{\eta})$ is

$$
\boldsymbol{J}(\boldsymbol{\eta})=\left[\begin{array}{ccc}
\cos \psi & -\sin \psi & 0 \\
\sin \psi & \cos \psi & 0 \\
0 & 0 & 1
\end{array}\right]
$$

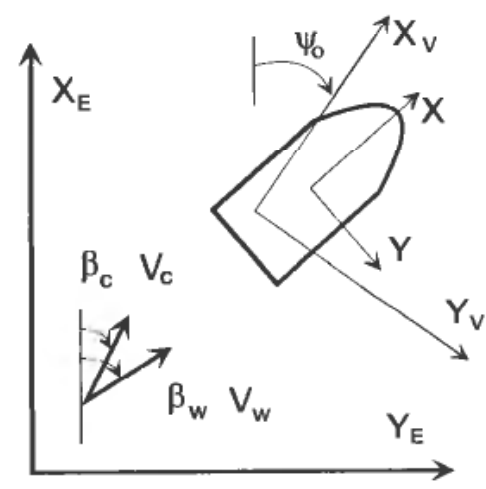

Figure 1. Reference frames. 


\subsection{Vessel dynamics}

The mathematical model describing the vessel dynamics is separated into a LF model and a WF model. The WF motions are assumed to be caused by first-order wave loads. Assuming small amplitudes these motions can be represented by a linear model. The LF motions are assumed to be caused by second-order mean and slowly varying wave loads, current loads, wind loads and thrust forces. These motions are generally nonlinear, but linear approximations around certain operation points can be found.

In the DP literature there has been some confusion related to the classification of energy dissipation in the WF and LF models. Mostly, linear damping is omitted in the derived LF models. In this paper linear damping proportional to the vessel velocities in surge, sway and yaw is introduced in both the LF model and the WF model. In addition nonlinear eddy-making damping proportional to the square of the velocity is included in the LF model. For decreasing velocities linear damping becomes more significant than nonlinear damping. An important contribution to linear damping in the LF model is viscous laminar skin friction and wave drift damping, which must not be mixed with the frequency dependent wave radiation damping used in the WF model, Faltinsen (1990). The effect of wave radiation damping is negligible in the LF model due to the low-frequency of oscillation.

Wave drift damping can be interpreted as added resistance for a ship advancing in waves and is according to Faltinsen and Sortland (1987) the most important damping contribution to the surge motion in higher sea states. The wave drift damping is proportional to the square of the significant wave height. There is also wave drift damping in sway and yaw. However, relative to the eddy-making damping the importance of the wave drift damping is small for the sway and yaw motion. Linear skin friction may be important for surge, especially for low sea states when the boundary layer is laminar. For higher sea states the viscous skin friction becomes turbulent and hence nonlinear, Faltinsen et al. (1986). Nonlinear damping due to eddy-making and turbulent skin friction is proportional to the square of the velocity and is often denoted as drag. The eddy-making damping is most important for sway and yaw motions. Theoretically the cross-flow principle and strip theory are used to calculate viscous damping in sway and yaw, while direct pressure integration is used to calculate eddy-making damping in surge. Both methods are semi-empirical in the sense that empirical drag coefficients are employed. Instead of using theoretical analysis, model tests are often used to calculate the damping

\subsubsection{Nonlinear low-frequency model}

The nonlinear body-fixed coupled equations of the LF motion in surge, sway and yaw are written

$$
M \dot{v}+C_{R B}(v) v+C_{A}\left(v_{r}\right) v_{r}+D_{L} v_{r}+D_{N L}\left(v_{r}, \gamma_{r}\right) v_{r}=\tau_{\text {wind }}+\tau_{\text {wave } 2}+\tau
$$

Here, $\tau$ is a control vector consisting of forces and moment in surge, sway and yaw provided by the thruster system. The effect of current is included in the relative velocity vector according to $v_{r}=\left[u-u_{c}, v-v_{c}, r\right]^{\mathrm{T}}$. The components of the current are defined as

$$
\begin{aligned}
u_{c} & =V_{c} \cos \left(\beta_{c}-\psi\right) \\
v_{c} & =V_{c} \sin \left(\beta_{c}-\psi\right)
\end{aligned}
$$

where $V_{c}$ and $\beta_{r}$ are the current velocity and direction respectively, see Fig. 1. Notice 
that current velocity in yaw is not considered. The mass matrix $M$ including added mass is defined as

$$
\boldsymbol{M}=\left[\begin{array}{ccc}
m-\boldsymbol{X}_{\dot{u}} & 0 & 0 \\
0 & m-\boldsymbol{Y}_{\dot{v}} & -\boldsymbol{Y}_{\dot{r}} \\
0 & -N_{\dot{v}} & \boldsymbol{I}_{z}-N_{r}
\end{array}\right]
$$

where $m$ is the vessel mass, $I_{z}$ is the moment of inertia about the $z$-axis. The zero-frequency added mass in surge, sway and yaw due to accelerations along the corresponding axes are defined as $X_{\dot{u}}<0, Y_{\dot{v}}<0$, and $N_{\dot{r}}<0$. Assuming small vessel velocity and starboard and port symmetries, the added mass in sway due to angular acceleration in yaw is equal to the added mass in yaw due to the sway acceleration, that is $Y_{\dot{r}}=N_{\dot{v}}$. Hence, the mass matrix is symmetrical and positive definite.

The skew-symmetric Coriolis and centripetal matrix of the rigid body is formulated

$$
C_{R B}(v)=\left[\begin{array}{ccc}
0 & 0 & -m v \\
0 & 0 & m u \\
m v & -m u & 0
\end{array}\right]
$$

The effect of current may be divided into two parts according to Wichers (1993); the potential part and the viscous part. The potential part of the current is modeled in a skew-symmetric Coriolis matrix, that is

$$
C_{A}(v)=\left[\begin{array}{ccc}
0 & 0 & Y_{\dot{v}} v_{r}+Y_{\dot{r}} r \\
0 & 0 & -X_{\dot{u}} u_{r} \\
-Y_{\dot{v}} v_{r}-Y_{\dot{r}} r & X_{\dot{u}} u_{r} & 0
\end{array}\right]
$$

The strictly positive damping matrix $D_{L}>0$, caused by linear wave drift damping and laminar skin friction damping is defined as

$$
\boldsymbol{D}_{L}=\left[\begin{array}{ccc}
-X_{u} & 0 & 0 \\
0 & -Y_{v} & -Y_{r} \\
0 & -N_{v} & -N_{r}
\end{array}\right]
$$

Based on model test the nonlinear damping vector is written

$$
\boldsymbol{D}_{N L}\left(v_{r}, \gamma_{r}\right) v_{r}=\left[\begin{array}{c}
0.5 \rho_{w} L_{p p} D C_{c x}\left(\gamma_{r}\right)\left|u_{r}\right| u_{r} \\
0.5 \rho_{w} L_{p p} D C_{c y}\left(\gamma_{r}\right)\left|v_{r}\right| v_{r} \\
0.5 \rho_{w} L_{p p}^{2} D C_{c \psi}\left(\gamma_{r}\right)\left|v_{r}\right| v_{r}
\end{array}\right]
$$

where $\rho_{w}$ is the density of water, $L_{p p}$ is the length between the ship perpendiculars, $D$ is the draft and $C_{c x}\left(\gamma_{r}\right), C_{c y}\left(\gamma_{r}\right)$ and $C_{c \psi}\left(\gamma_{r}\right)$ are the non-dimensional drag coefficients in surge, sway and yaw respectively. The drag coefficients are found by model testing.

The relative drag angle is found from the following relation

$$
\gamma_{r}=\operatorname{atan} 2\left(v_{r}, u_{r}\right)
$$

The effect of wind may he divided into mean, slowly-varying and rapidly-varying wind 
loads. For DP applications only mean and slowly varying wind loads are considered. The wind velocity is assumed to be much larger than the vessel velocity such that the components of the wind velocities are defined according to

$$
\begin{aligned}
& u_{w}=V_{w} \cos \left(\beta_{w}-\psi\right) \\
& v_{w}=V_{w} \sin \left(\beta_{w}-\psi\right)
\end{aligned}
$$

where $V_{w}$ and $\beta_{w}$ are the wind velocity and direction respectively, see Fig. 1. The wind load vector in surge, sway and yaw are written

$$
\tau_{\text {wind }}=\left[\begin{array}{c}
0.5 \rho_{a} A_{w x} C_{w x}\left(\gamma_{w}\right)\left|u_{w}\right| u_{w} \mid \\
0.5 \rho_{a} A_{w y} C_{w y}\left(\gamma_{w}\right)\left|v_{w}\right| v_{w} \\
0.5 \rho_{a} A_{w y} L_{o a} C_{w \psi}\left(\gamma_{w}\right)\left|v_{w}\right| v_{w}
\end{array}\right]
$$

where $\rho_{a}$ is the density of air, $L_{o a}$ is the overall length of the vessel, $A_{w x}$ and $A_{w y}$ are the lateral and longitudinal areas of the non-submerged part of the ship projected on the xz-plane and yz-plane. $\boldsymbol{C}_{w x}\left(\gamma_{w}\right), \boldsymbol{C}_{w y}\left(\gamma_{w}\right)$ and $\boldsymbol{C}_{w \psi}\left(\gamma_{w}\right)$ are the non-dimensional wind coefficients in surge, sway and yaw respectively. These coefficients are often found by model testing or by employing semi-empirical formulas as presented in Isherwood (1972). The relative wind angle is defined as

$$
\gamma_{w}=\beta_{w}-\psi
$$

The wave drift loads contribute to a significant part of the total excitation force in the LF model. The determination of the second-order wave effects can be done by means of quadratic transfer functions, Faltinsen (1990). However, this is time consuming and requires a large amount of input data. An approximate approach proposed by Newman (1974) using frequency dependent wave drift coefficients may be applied. By dividing the sea wave spectrum (usually of Pierson-Moskowitz type) in $N$ equal frequency intervals with corresponding wave frequency $\omega_{j}$ and amplitude $A_{j}$, the wave drift loads for surge $(i=1)$, sway $(i=2)$ and yaw $(i=3)$ are found to be

$$
\tau_{\mathrm{wave} 2}^{i}=\bar{\tau}_{w m}^{i}+\tau_{w s v}^{i}=\sum_{j=1}^{N} A_{j}^{2} T_{j j}^{i}\left(\omega_{j}\right)+2\left(\sum_{j=1}^{N} A_{j}\left(T_{j j}^{i}\left(\omega_{j}\right)\right)^{1 / 2} \cos \left(\omega_{j} t+\varepsilon_{j}\right)\right)^{2}
$$

where $T_{j j}^{i}\left(\omega_{j}\right)>0$ is the frequency dependent wave drift function and $\varepsilon_{j}$ is a random phase angle. In general the second-order wave loads are much less than the first-order wave loads. The second-order wave loads are proportional to the square of the wave amplitude, whereas the first-order wave loads are proportional to the wave amplitude. This means that the second-order wave loads have increased importance for increasing sea state, at least up to a certain sea state.

\subsubsection{Linear low-frequency model}

For the purpose of controller design, it is convenient to apply a linear LF model around zero relative vessel velocity. In order to reduce the coupling in the rotation matrix the vessel position and heading angle are referred to the vessel-parallel frame. Assuming small yaw rotations about the desired heading angle $\Delta \psi=\psi-\psi_{0}$, the rotation matrix can be approximated by the identity matrix. The measured position and heading signals must be transformed to the vessel-parallel frame hefore they are 
processed by the estimator and controller. The linear LF state space model can be formulated as

$$
\begin{aligned}
& \dot{x}_{L}=A_{L} x_{L}+B_{\Lambda} \tau+\Gamma_{L} w_{L} \\
& y_{L}=C_{L} x_{L}+v
\end{aligned}
$$

The state space vector is defined as $x_{L}=\left[v^{\mathrm{T}}, \eta^{\mathrm{T}}\right]^{\mathrm{T}}$, where for notation simplicity the incremental symbol $\Delta$ is omitted. $w_{L}$ is a 3 -dimensional disturbance vector consisting of current, wind and wave drift loads. $y_{L}$ is a 3-dimensional measurement vector containing the surge and sway positions, and the yaw angle. $v$ is a 3 -dimensional vector of sensor noise which is assumed to be Gaussian white noise. The $6 \times 6$ system matrix is then written

$$
A_{L}=\left[\begin{array}{cc}
-M^{-1} D_{L} & 0_{3 \times 3} \\
I_{3 \times 3} & 0_{3 \times 3}
\end{array}\right]
$$

where $I_{3 \times 3}$ is the $3 \times 3$ identity matrix and $0_{3 \times 3}$ is the $3 \times 3$ zero matrix. The $6 \times 3$ control input matrix is independent of the actual thruster configuration and becomes

$$
B_{L}=\left[\begin{array}{c}
M^{-1} \\
0_{3 \times 3}
\end{array}\right]
$$

The $6 \times 3$ disturbance input matrix is

$$
\Gamma_{L}=\left[\begin{array}{c}
M^{-1} \\
0_{3 \times 3}
\end{array}\right]
$$

Converting the position and heading measurements to the vessel-parallel frame, the $3 \times 6 \mathrm{LF}$ measurement matrix becomes

$$
C_{L}=\left[0_{3 \times 3} \quad I_{3 \times 3}\right]
$$

\subsubsection{Linear wave-frequency model}

Since it is possible to obtain results in irregular seas by linearly superposing results from regular wave components, it is sufficient to analyse the vessel in regular sinusoidal waves of small steepness. The hydrodynamic problem in regular waves is solved as two sub-problems which are added together to give the total linear wave induced loads (Faltinsen, 1990).

- Wave reaction: Forces and moments on the vessel when the vessel is forced to oscillate with the wave excitation frequency. The hydrodynamic loads are identified as added mass and wave radiation damping terms.

- Wave excitation: Forces and moments on the vessel when the vessel is restrained from oscillating and there are incident waves. This gives the wave excitation loads which are composed of so-called Froude-Kriloff (undisturbed pressure field) and diffraction forces and moments (changes in undisturbed pressure field).

The coupled equations of WF motion in surge, sway and yaw are assumed to be linear and can, in the vessel-parallel frame, be formulated as

$$
M(\omega) \ddot{\eta}_{W}+D_{p}(\omega) \dot{\eta}_{W}=\tau_{\text {wavel }}
$$

where the WF motion vector for surge and sway translations and yaw rotations is defined 
as $\eta_{W}=\left[\eta_{1}, \eta_{2}, \eta_{6}\right]^{\mathrm{T}} . \tau_{\text {wavel }}$ is a 3 -dimensional excitation vector which will be modified for varying vessel heading relative to the incident wave direction.

$M(\omega)$ is a $3 \times 3$ mass matrix containing frequency depending added mass coefficients in addition to the vessel mass and moment of inertia. $D_{p}(\omega)$ is a $3 \times 3$ wave radiation damping matrix. An important feature of the added mass terms and the wave radiation (potential) damping terms are the memory effects or the frequency dependence. This can be taken into account by introducing a convolution integral or a so-called retardation function, Newman (1977). The structure of the mass and damping matrices are the same as in (5) and (8).

The WF response spectra of the surge and sway translations and the yaw rotations can in irregular seas be found by reformulating (20) as a transfer function matrix and by representing the wave loads by wave load spectra. These response spectra are often in controller design approximated by synthetic white noise driven processes consisting of three uncoupled harmonic oscillators with damping (Sælid et al. 1983). In state space form a synthetic WF model can be written

$$
\begin{aligned}
& \dot{x}_{W}=A_{W} x_{W}+\Gamma_{W}{ }^{\mathrm{n}} \\
& y_{W}=C_{W} x_{W}
\end{aligned}
$$

where the state space vector is defined as $x_{W}=\left[\eta_{W}^{\mathrm{T}}, \xi^{\mathrm{T}}\right]^{\mathrm{T}} . y_{W}$ is a 3 -dimensional measurement vector of the WF surge and sway positions, and the WF yaw angle and $n$ is white noise. The $6 \times 6$ system matrix is derived to be

$$
A_{W}=\left[\begin{array}{cc}
A_{W}^{11} & A_{W}^{21} \\
I_{3 \times 3} & 0_{3 \times 3}
\end{array}\right]
$$

where

$$
\begin{aligned}
A_{W}^{11} & =\left[\begin{array}{ccc}
-2 \zeta_{1} \omega_{1} & 0 & 0 \\
0 & -2 \zeta_{2} \omega_{2} & 0 \\
0 & 0 & -2 \zeta_{6} \omega_{6}
\end{array}\right] \\
A_{W}^{21} & =\left[\begin{array}{ccc}
-\omega_{1}^{2} & 0 & 0 \\
0 & -\omega_{2}^{2} & 0 \\
0 & 0 & -\omega_{6}^{2}
\end{array}\right]
\end{aligned}
$$

where $\zeta_{i}$ is the relative damping ratio and $\omega_{i}$ is the natural frequency. The relative damping ratio $\zeta_{i}$ is often in the range of $0.05-0 \cdot 2$, while the resonance frequency $\omega_{i}$ is related to the bandwidth of the WF model, see Sagatun et al. (1995) for details. The $6 \times 1$ disturbance matrix is written

$$
\Gamma_{W}=\left[k_{1}, k_{2}, k_{3}, 0,0,0\right]^{\mathrm{T}}
$$

where $k_{1}, k_{2}$ and $k_{3}>0$ are the covariance of the white noise process reflecting the WF response caused by the actual sea state. $k_{1}, k_{2}$ and $k_{3}$ are linear dependent of the significant wave height. The $3 \times 6$ measurement matrix is written

$$
C_{W}=\left[\begin{array}{ll}
I_{3} \times 3 & 0_{3 \times 3}
\end{array}\right]
$$

The sensor noise in the WF model is omitted since it is already modeled in the LF model. 


\subsection{Linear thruster model}

Usually, perfect control action without imposing the thruster dynamics are assumed. Unfortunately, this will not be true in the real system. The thrust response is affected by the dynamics in the actuators and the drive system. This will cause reduced command following capabilities such as phase lag and amplitude reduction when the frequency increases. There will also be a loss of thrust efficiency due to disturbances in the water inflow to the thruster blades caused by thrust-to-thrust and thrust-to-hull interactions, current and vessel velocities. In addition the influence from the free surface will affect the thrust efficiency. Reduced thruster efficiency caused by disturbances in the water inflow is compensated for in the thrust allocation algorithm. While, the dynamics in the actuator and drive systems will be accounted for in the controller. Experience obtained from full-scale experiments indicates that a first-order model is well suited for this purpose. Hence, the thruster dynamics is represented by the following model

$$
\dot{\tau}=-A_{t r} \tau+A_{t r} \tau_{c}
$$

where $\tau_{c}$ is the commanded equivalent thrust force vector. The $3 \times 3$ diagonal thruster dynamics matrix is defined as

$$
A_{t r}=\operatorname{diag}\left(\frac{1}{T_{t r 1}}, \frac{1}{T_{t r 2}}, \frac{1}{T_{t r 3}}\right)
$$

Here, $T_{t r 1}, T_{t r 2}$ and $T_{t r 3}$ are the equivalent thruster time constants in surge, sway and yaw. For conventional ships $T_{t r 1}$ can be approximated by the time constants corresponding to the main propellers. $T_{t r 2}$ and $T_{t r 3}$ can be approximated by the time constants to the tunnel and azimuth thrusters. Moreover, it is reasonable to assume that $T_{t r 2} \approx T_{t r 3}$.

\section{DP controller design}

The DP controller consists of a feedback controller and a feedforward controller. The proposed feedback control loop is inspired by the work of Balchen (1993) and is modified for the purpose of DP application.

The computation of feedback signals to be used in the controller are based on Kalman filter and observer theory and are described in Sagatun $\boldsymbol{e t}$ al. (1995).

\subsection{Control design plant model}

In order to produce the desired control action a design plant model to be used in conjunction with the controller is derived according to

$$
\hat{x}=A \hat{x}+B \tau_{c}
$$

where the 9-dimensional estimated state space vector is defined as $\hat{\boldsymbol{x}}=\left[\hat{\boldsymbol{x}}_{L}^{\mathrm{T}}, \hat{\tau}\right]^{\mathrm{T}}$. The $9 \times 9$ augmented system matrix is written

$$
\boldsymbol{A}=\left[\begin{array}{cc}
A_{L} & B_{L} \\
0_{3 \times 3} & -A_{t r}
\end{array}\right]
$$

The $9 \times 9$ control input matrix $\boldsymbol{B}$ becomes

$$
\boldsymbol{B}=\left[\begin{array}{c}
0_{6 \times 3} \\
A_{t r}
\end{array}\right]
$$




\subsection{Reference model}

For DP operations close to other offshore structures or ships, it is crucial to be capable to perform controlled movements and rotations of the vessel. The automatic guidance function which takes the vessel from the prevailing coordinates to the new setpoint is defined as marked position and can be specified either in the vessel-parallel frame or in the earth-fixed frame. The movement and rotation can be done for each degree of freedom separately or as a fully 3 DOF coupled motion. In order to provide high performance DP operations with bumbles transfer between station-keeping and marked position operations, a reference model is introduced for calculation of feasible trajectories of the desired vessel motion for each degree of freedom. Experience achieved from full-scale experiments have demonstrated that the following reference model in the earth-fixed frame is appropriate (Fossen 1994)

$$
a_{d}^{e}+\Omega v_{d}^{e}+\Gamma x_{d}^{e}=\Gamma x_{\mathrm{ref}}^{e}
$$

The 3-dimensional vectors $a_{d}^{e}, v_{d}^{e}$ and $x_{d}^{e}$ define the desired vessel acceleration, velocity and position trajectories in the earth-fixed frame. The vector $x_{\text {ref }}^{e}$ defines the new reference coordinates either relative to the previous setpoint or as global earth-fixed coordinates. The design parameters in the reference model consist of a $3 \times 3$ diagonal damping matrix $\Omega>0$ and a $3 \times 3$ diagonal stiffness matrix $\Gamma>0$. The earth-fixed desired vessel acceleration, velocity and position trajectories are transformed into a moving vessel-parallel frame which follows the desired earth-fixed position and heading trajectory. Hence

$$
\begin{aligned}
& a_{d}=J^{T} a_{d}^{e} \\
& v_{d}=J^{T} v_{d}^{e} \\
& x_{d}=J^{T} x_{d}^{e}
\end{aligned}
$$

The reference model will also run in station-keeping operations, but then with the desired velocity and acceleration equal to zero.

\subsection{LQG control law}

The proposed LQG algorithm takes the deviation vector between the state estimate vector and the desired state vector and produces a proportional feedback to the control vector. Assuming that the nominal vessel and thruster model is detectable and stabilizable, the LQG feedback control law is derived by minimizing the following performance index

$$
J=E\left\{\lim _{T \rightarrow \infty} \frac{1}{T} \int_{0}^{T}\left(e^{T} Q e+\tau_{L Q}^{T} P \tau_{L Q}\right) d t\right\}
$$

The deviation vector is defined as

$$
e=\left[e_{1}^{T}, e_{2}^{T}, e_{3}^{T}\right]^{\mathrm{T}}
$$

where

$$
\begin{aligned}
& e_{1}=[\hat{u}, \hat{v}, \hat{r}]^{\mathrm{T}} \\
& e_{2}=[\hat{x}, \hat{y}, \hat{\psi}]^{\mathrm{T}} \\
& e_{3}=\hat{\tau}-\tau_{F F}-\tau_{I}-\tau_{d}
\end{aligned}
$$


Here, $\hat{t}$ is the estimated thruster forces and moment vector in surge, sway and yaw. $Q=Q^{\mathrm{T}} \geqslant 0$ is a $9 \times 9$ error weighting matrix and $P=P^{\mathrm{T}}>0$ is a $3 \times 3$ control weighting matrix. By minimizing the control index $J$, the LQG method returns the control gain matrix $G_{L Q}$. For linear time invariant systems stationary solutions of the Ricatti equation can be found. Hence, the Ricatti equation computations reduce to solving

$$
-A R_{\circledast}-A^{\mathrm{T}} R_{\infty}+R_{\infty} B P^{-1} B^{\mathrm{T}} R_{\infty}-Q=0
$$

where $R_{\infty}$ is a $9 \times 9$ non-negative symmetric matrix.

The $3 \times 9$ stationary LQG control gain matrix is

$$
G_{L Q}=P^{-1} B^{\mathrm{T}} R_{\infty}
$$

The LQG control law is then

$$
\tau_{L Q}=-G_{L Q} e
$$

\subsection{Integral action}

In order to meet the special command following and disturbance rejection performance specifications, it is necessary to append three free integrators to the control model. It is then desirable to define a property space $z \in \mathfrak{R}^{3}$, which expresses the variables to be controlled towards certain set points, Balchen (1993). The dimension of the property space is the same as the control vector space, that is $\tau \in \mathfrak{R}^{3}$. The certain variables to be controlled to the set points are fewer than those constituting the state space $e \in \mathfrak{R}^{9}$. The relation between these variables and the state space is given through the transformation

$$
z=g(e)=e_{2}
$$

A multivariable PI algorithm is then achieved by means of an integral loop controlling the property $z$ in parallel with the proportional LQ control loop. Inspired from classical SISO PI controller tuning one can specify the eigenvalues of the integral loop in a diagonal matrix $\Lambda_{G}$. Since the integral loop will be slower than the proportional LQ loop, the following approximation for the integral loop can be found

$$
\dot{z}=G_{I} G_{z}\left(A-B G_{L Q}\right)^{-1} B z=\Lambda_{G} z
$$

where $G_{z}$ is a $3 \times 9$ property matrix given by

$$
G_{z}=\frac{\partial g(e)}{\partial e}=\left[\begin{array}{lll}
0_{3 \times 3} & I_{3 \times 3} & 0_{3 \times 3}
\end{array}\right]
$$

This leads to the $3 \times 3$ integral loop gain matrix

$$
G_{1}=\Lambda_{G}\left(G_{2}\left(A-B G_{L Q}\right)^{-1} B\right)^{-1}
$$

where the matrix $G_{z}\left(A-B G_{L Q}\right)^{-1} B$ must be non-singular. Open integrators as they appear both in the control loop and the estimator are equipped with anti-windup precautions in case their outputs reach amplitude restrictions such as saturation in the physical control devices. The resulting integral control law is written

$$
\dot{\tau}_{I}=A_{W} \tau_{I}+G_{I} z
$$

where $A_{W}$ is a $3 \times 3$ anti windup precaution matrix. 


\subsection{Wind feedforward controller}

Wind loads have an important impact on the vessel response. In order to obtain fast disturbance rejection with respect to varying wind loads, it is desirable to introduce a wind feedforward controller. The wind forces and moment in surge, sway and yaw are estimated by a Kalman filter and a Luenberger observer, where the wind velocity and wind direction are measured. The estimates are multiplied by a feedforward gain matrix. The wind feedforward control law is taken to be

$$
\tau_{F F}=-G_{W} \hat{\tau}_{\text {wind }}
$$

where $\hat{\tau}_{\text {wind }}$ is the estimated wind forces and moment in surge, sway and yaw respectively produced by a wind force estimator. It is assumed that the wind loads are uncoupled, such that the gain matrix $G_{W}$ is a $3 \times 3$ diagonal matrix

$$
G_{W}=\operatorname{diag}\left(g_{w 1}, g_{w 2}, g_{w 3}\right)
$$

where $0 \leqslant g_{w i} \leqslant 1$ for $i=1 \ldots 3$.

\subsection{Model reference feedforward controller}

In order to improve the performance of the controller during marked position (tracking) operations a feedforward control action based on input from the reference model is included. The feedforward control action is derived to be

$$
\tau_{d}=M a_{d}+D_{L} v_{d}+C_{R B}\left(v_{d}\right) v_{d}+C_{A}\left(v_{d}\right) v_{d}
$$

\subsection{Resulting DP control law}

The resulting DP control law is found by adding (36), (41), (42) and (44), that is

$$
\tau_{c}=\tau_{L Q}+\tau_{I}+\tau_{F F}+\tau_{d}
$$

\subsection{Optimal thrust allocation}

The relation between the equivalent control vector $\tau_{c}$ and the actual thruster action $u_{c}$ are defined according to

$$
\tau_{c}=T K u_{c}
$$

where $T$ is a $3 \times r$ thrust configuration matrix and $r$ is the number of thrusters. $K$ is an $r \times r$ diagonal matrix of thrust force coefficients (Fossen et al. 1995) and $u_{c}$ is the actual control vector of either pitch controlled or revolution controlled propeller inputs. If the number of control inputs $r$ is not equal to the number of outputs (surge, sway and yaw) to be controlled, the thrust configuration matrix becomes singular and the inverse matrix cannot be found. Then the inverse thrust configuration matrix $T^{-1}$ is replaced with the generalized inverse $T^{+}$. This matrix may be found by minimizing the quadratic energy cost function according to

$$
\min J=\frac{1}{2}\left(K u_{c}\right)^{\mathrm{T}} W\left(K u_{c}\right)
$$

subject to

$$
\tau_{c}-T K u_{c}=0
$$

where $W$ is an $r \times r$ positive definite matrix providing mutual weighting of the different thrusters with respect to minimum control energy. $W$ is usually diagonal and the diagonal elements should be selected such that the control actions are directed towards 
the desired thruster. Large element in $W$ means that the actual thruster is expensive to use. The solution of the optimization problem is written

$$
T^{+}=W^{-1} T^{\mathrm{T}}\left(T W^{-1} T^{\mathrm{T}}\right)^{-1}
$$

Hence, the commanded control action provided by the actual thrusters becomes

$$
u_{c}=K^{-1} T^{+} \tau_{c}
$$

\section{Full-scale results}

The DP system has been implemented by using ABB Master and Advant products. Experience obtained from commercial installations of the ABB DP system, have successfully demonstrated the performance of the proposed controller.

In this paper results from full-scale experiments with the multipurpose supply vessel, Northern Clipper owned by Saevik Supply Management, are shown. The installed ABB DP system configuration and Northern Clipper are illustrated in Figs 7 and 8 in Appendix A. The main dimensions of the vessel are: design draught is $6.25 \mathrm{~m}$, vessel dead-weight at draught $6.25 \mathrm{~m}$ is 4200 tons, length between the perpendiculars is $82.5 \mathrm{~m}$. Northern Clipper is equipped with two main propellers, two stern tunnel thrusters, one bow tunnel thruster and one compass thruster.

The main engine consists of two $3530 \mathrm{~kW}$ Ulstein Bergen engines. Three different types of position reference systems are installed; differential GPS, taut wire and the underwater based hydroacoustic position reference (HPR) system.

In Figs 2-6 time series recorded on Northern Clipper are shown. All time series were recorded during the same DP operation. The vessel was operated in station-keeping function in $60 \mathrm{~s}$. Then the operator changed the setpoint and ordered the vessel to move $18 \mathrm{~m}$ to the south and $19 \mathrm{~m}$ to the east without changing the heading. After about $300 \mathrm{~s}$

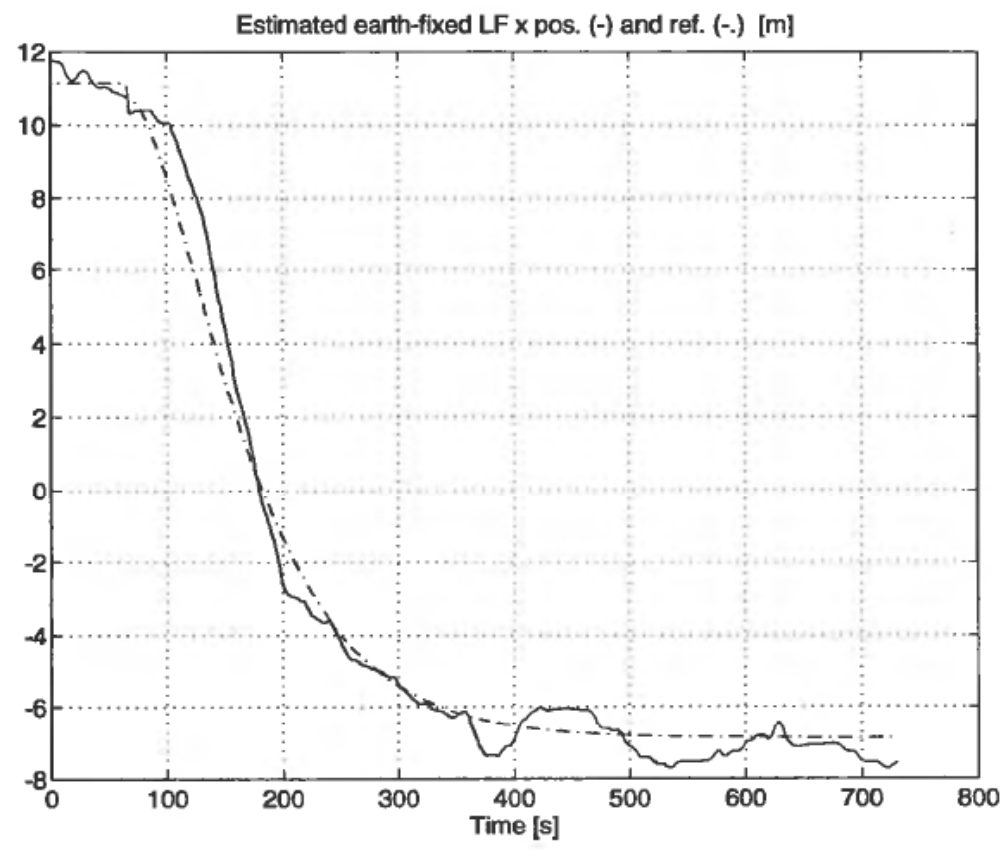

Figure 2. Estimated (- - ) and desired (-.-) earth-fixed $x$ position during station-keeping and marked position operations with $\Delta x=-18 \mathrm{~m}, \Delta y=19 \mathrm{~m}$ and $\Delta \psi=0 \mathrm{deg}$. 


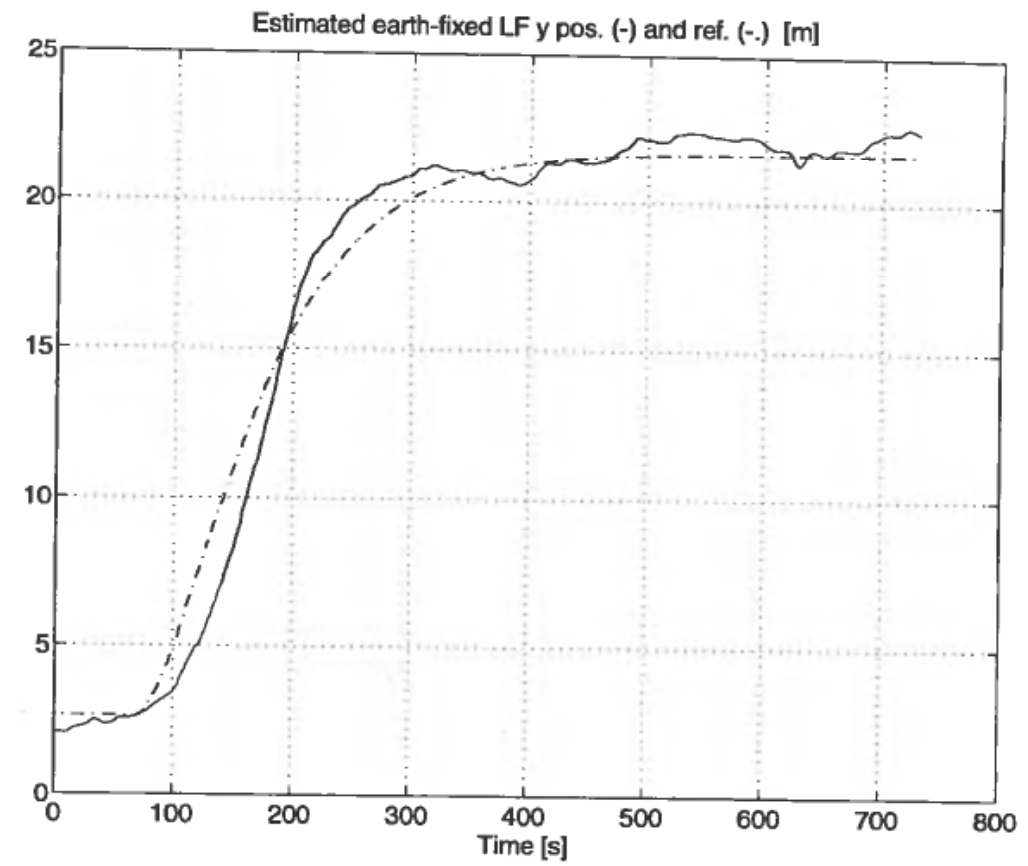

Figure 3. Estimated (- - ) and desired (-.-) earth-fixed $y$ position during station-keeping and marked position operations with $\Delta x=-18 \mathrm{~m}, y=19 \mathrm{~m}$ and $\Delta \psi=0 \mathrm{deg}$.

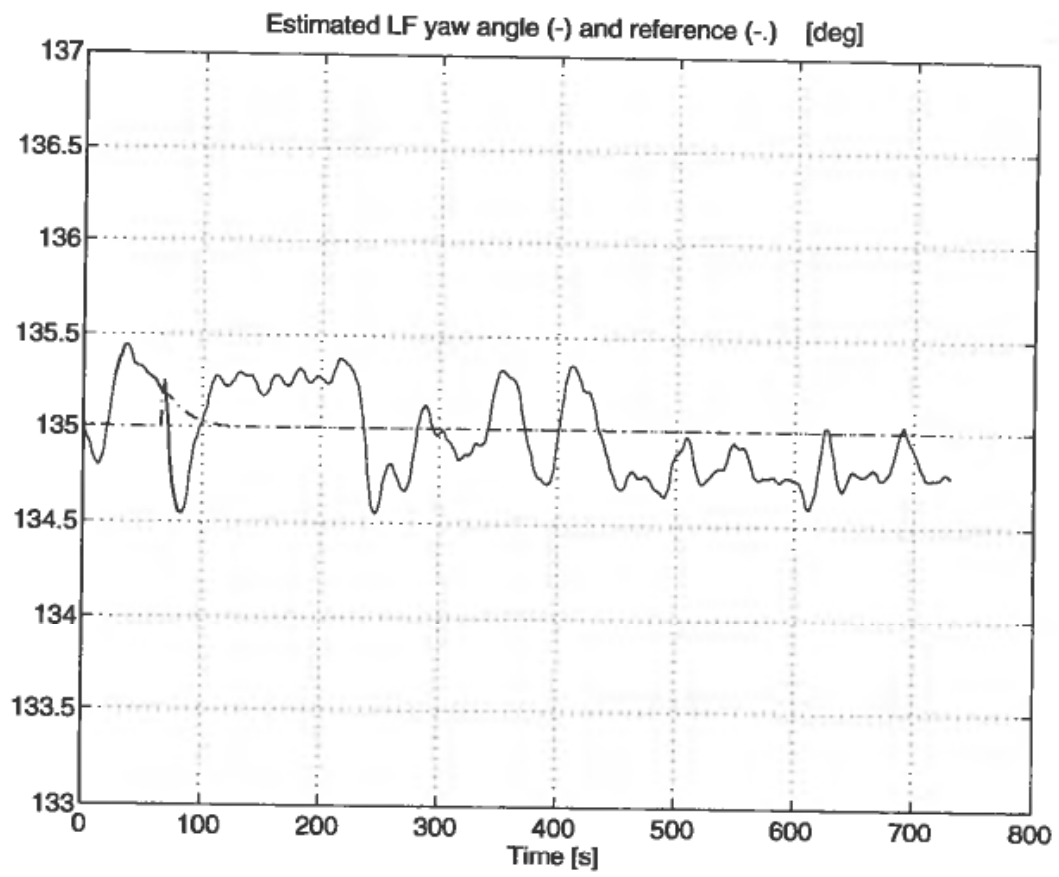

Figure 4. Estimated (- - ) and desired (-.-) heading angle $\psi$ angle during station-keeping and marked position operations with $\Delta x=18 \mathrm{~m}, \Delta y=19 \mathrm{~m}$ and $\Delta \psi=0 \mathrm{deg}$. 


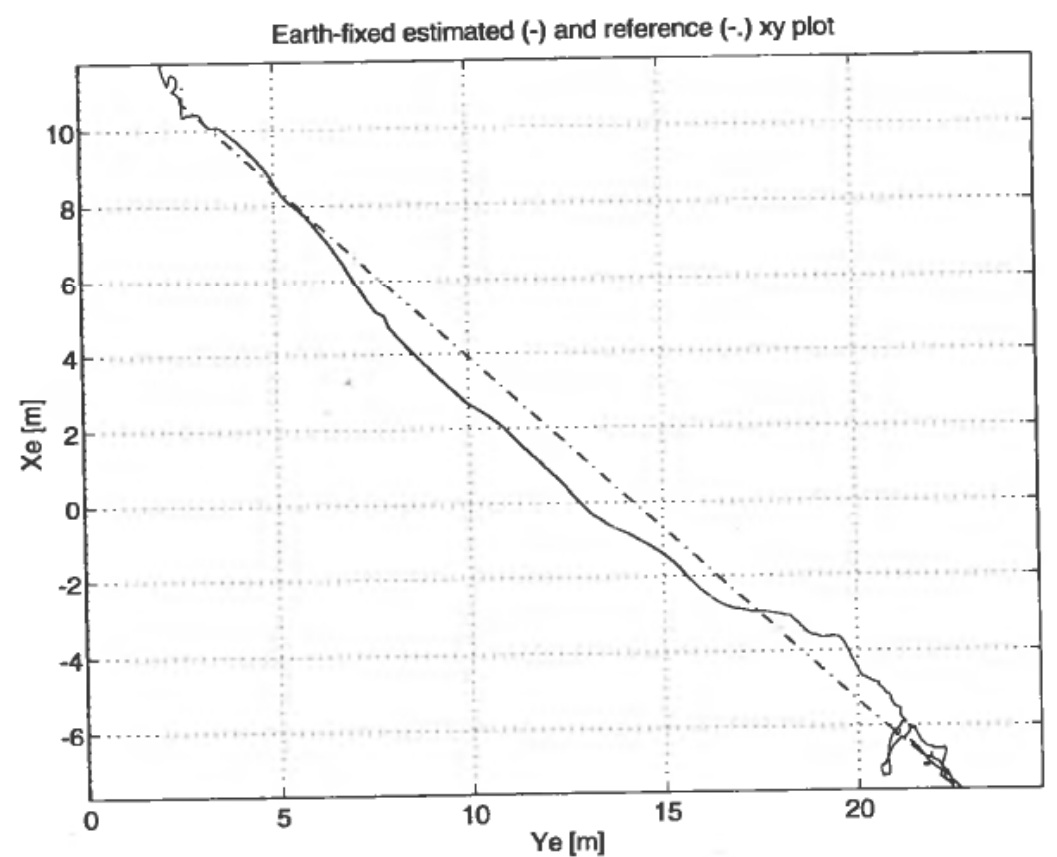

Figure 5. Estimated (-) and desired (-.-) $x y$-plot of marked position and station-keeping operations with $\Delta x=-18 \mathrm{~m}, \Delta y=19 \mathrm{~m}$ and $\Delta \psi=0$ deg.
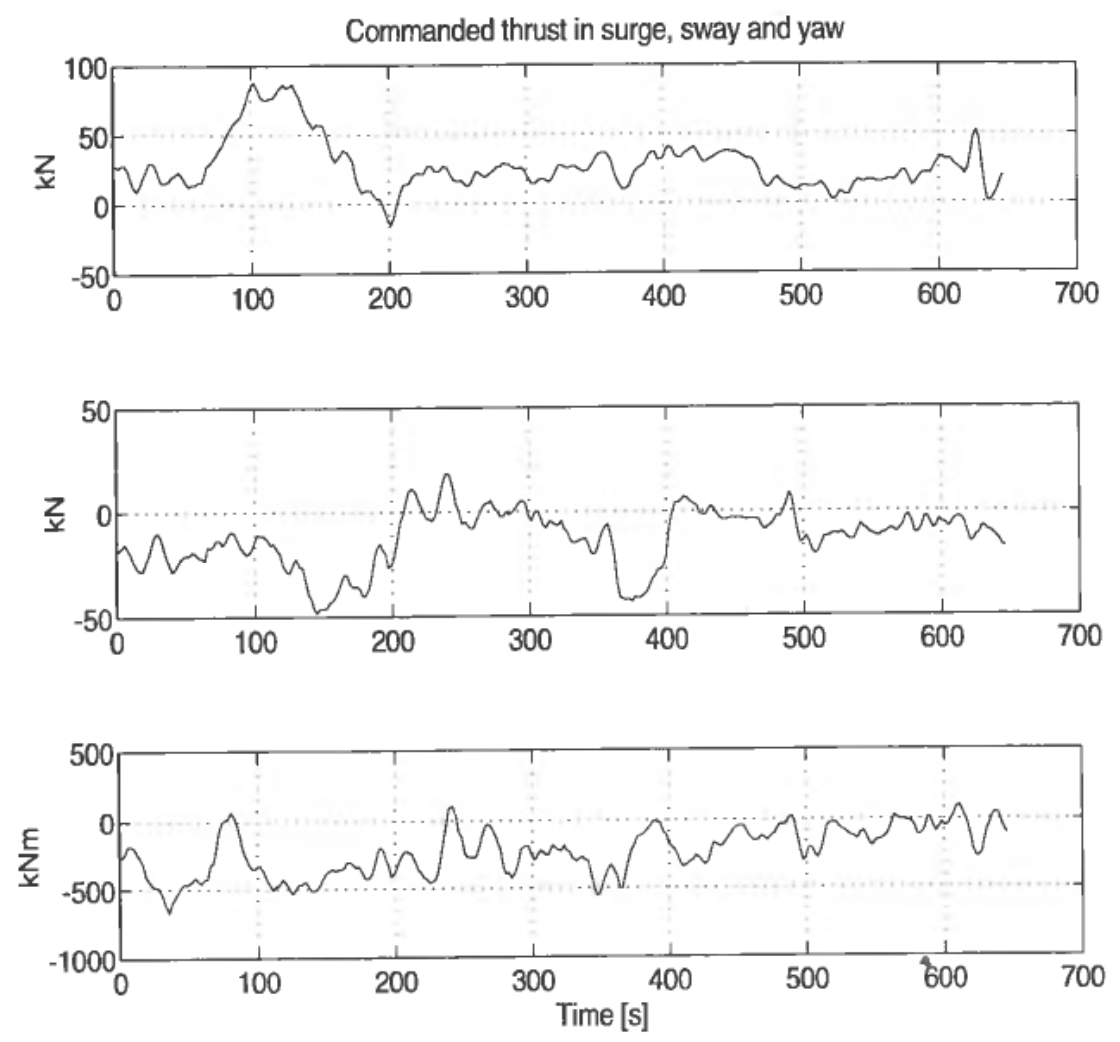

Figure 6. Commanded thrust during station-keeping and marked position operations with $\Delta x=-18 \mathrm{~m}, \Delta y=19 \mathrm{~m}$ and $\Delta \psi=0 \mathrm{deg}$. 
the vessel reached the new desired position and then the DP system automatically returned to station-keeping function. The significant wave height was about $1 \mathrm{~m}$ and the mean wind velocity was $10 \mathrm{~m} / \mathrm{s}$ with zero relative direction. Taut wire and DGPS were used as reference systems during these operations.

\section{Conclusions}

A dynamic positioning (DP) system using model-based control is proposed. Commercial DP systems have different control modes for automatic positioning and guidance of marine vessels by means of thruster and propeller actions. This paper describes a control algorithm which provides bumbles transfer between station-keeping and tracking (marked position) operations. A reference model is used to provide appropriate reference trajectories. Since it is not desirable to counteract the wave-frequency movement caused by first-order wave loads, the control action of the propulsion system is produced by the low-frequency part of the vessel movement caused by current, wind and second-order mean and slowly varying wave loads. Kalman filter theory is used for state estimation. Full-scale experiments with a supply vessel have successfully demonstrated the performance of the proposed controller.

\section{ACKNOWLEDGEMENTS}

ABB Industri AS, ABB Corporate Research Norway, Saevik Supply Management AS and Norsk Hydro AS are gratefully acknowledged for fruitful cooperation and contributions. Parts of this work were carried out when the first author was Research Scientist at ABB Corporate Research Norway.

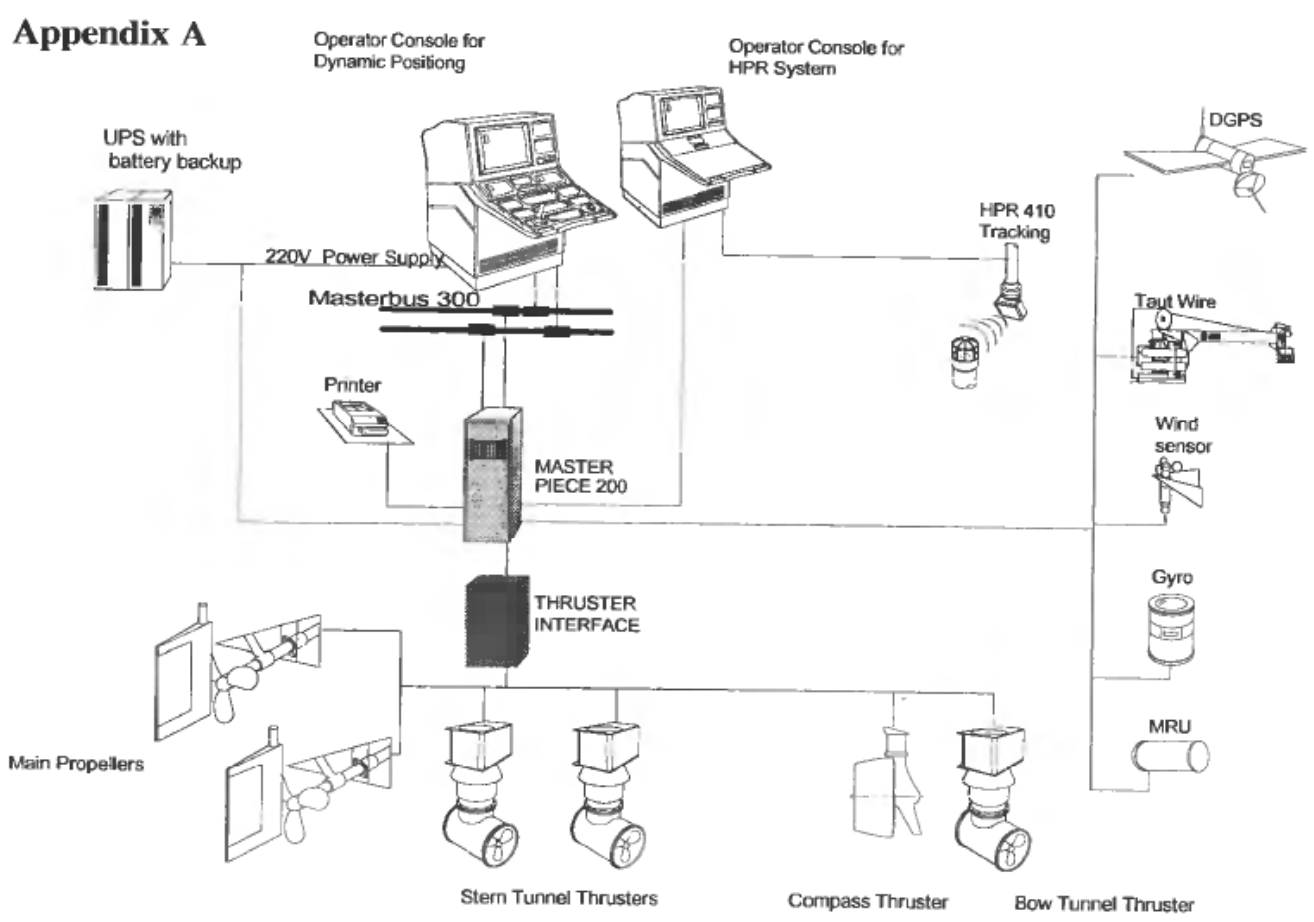

Figure 7. The ABB DP system configuration for Northern Clipper. 


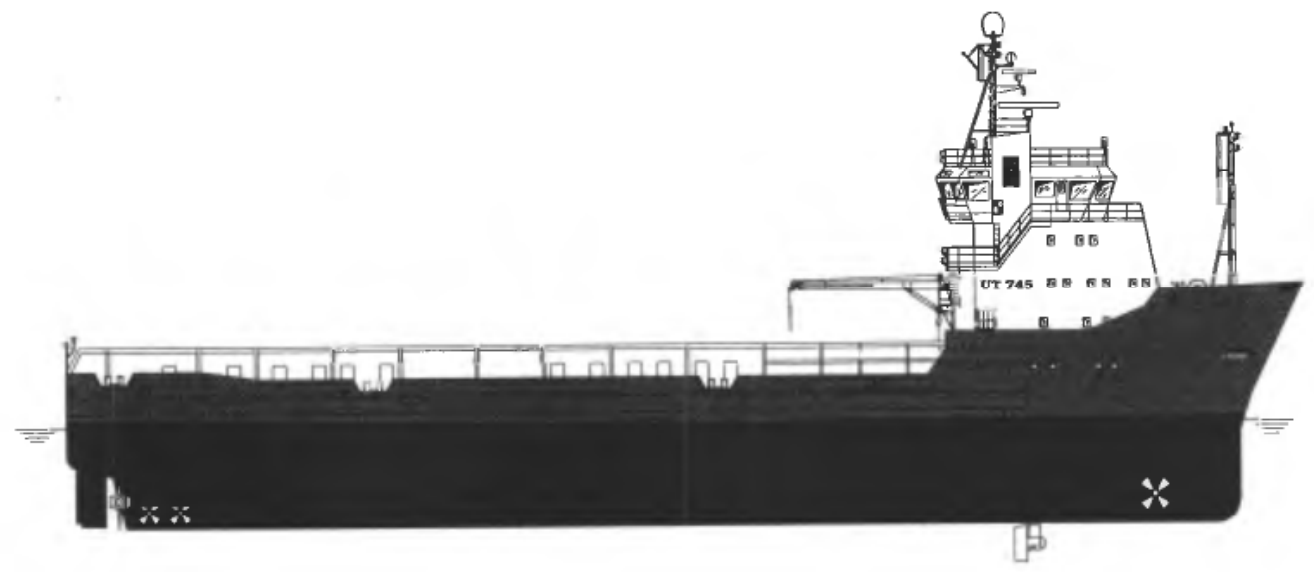

Figure 8. Northern Clipper owned by Sævik Supply Management.

\section{REFERENCES}

BALCHEN, J. G. (1993). A modified LQG algorithm (MLQG) for robust control of nonlinear multivariable systems. Modeling, Identification and Control, 14, pp. 175-180.

BALCHEN, J. G., JENSSEN, N. A. and SALID, S. (1976). Dynamic positioning using Kalman filtering and optimal control theory. IFAC/IFIP Symp. on Automation in Offshore Oil Field Operation, Holland, Amsterdam, pp. 183-186.

Balchen, J. G., JensSEN, N. A. and SÆLID, S. (1980a). Dynamic positioning of floating vessels based on Kalman filtering and optimal conurol. Proc. of the 19th IEEE Conf. on Decision and Control, Albuquerque, N.M., pp. 852-864.

BALCHEN, J. G., JeNSSEN, N. A. and S FlLID, S. (1980b). A dynamic positioning system based on Kalman filtering and optimal control. Modeling, Identification and Control, 1, pp. 135-163.

Faltinsen, O. M., DAhle, L. and Sortland, B. (1986). Slowdrift damping and response of a moored ship in irregular waves. Proc. 3rd OMAE Conf., Tokyo, Japan.

Faltinsen, O. M. and SorTland, B. (1987). Slow drift eddy making damping of a ship. Applied Ocean Research, 9, 1, pp. 37-46.

FALTINSEN, O. M. (1990). Sea Loads on Ships and Offshore Structures (Cambridge University Press).

FOSSEN, T. I. (1994). Guidance and Control of Ocean Vehicles (John Wiley and Sons Ltd).

FosSEN, T. I., SAGATUN, S. I. and SøRENSEN, A. J. (1995). Identification of dynamically positioned ships. Proc. of the 3rd IFAC Workshop on Control Applications in Marine Systems (CAMS'95), Trondheim, Norway.

Fung, P. T-K. and GrimBle, M. (1983). Dynamic ship positioning using self-tuning Kalman filter. IEEE Trans. on Automatic Control, AC-28, pp. 339-349.

Grimble, M. J., PATton, R. J. and Wise, D. A. (1980a). The design of dynamic ship positioning control systems using stochastic optimal control theory. Optimal Control Applications and Methods, 1, pp. 167-202.

Grimble, M. J., PATTON, R. J. and WiSE, D. A. (1980b). Use of Kalman filtering techniques in dynamic ship positioning systems. IEE Proc., 127, D, pp. 93-102.

IsHERWOOD, M. A. (1972). Wind resistance of merchant ships. Trans. Inst. Naval Arch., RINA, 115, pp. 327-338.

Newman, J. N. (1974). Second order slowly varying forces on vessels in irregular waves. Proc. Int. Symp. on the Dynamics of Marine Vehicles and Structures in Waves, London, England, Mechanical Engineering Publications Ltd. pp. 182-186.

Newman, J. N. (1977). Marine Hydrodynamics (MIT Press, Cambridge, Massachusetts).

Sagatun, S. I., Sørensen, A. J. and Fossen. T. I. (1995). State estimation and dynamic positioning of marine vessels. The paper is submitted to the IEEE Trans. on Control Systems Technology (1995).

Salvesen, N., TuCK, E. O. and FAltinsen, O. M. (1970). Ship motions and seal loads. Trans. SNAME, 78, pp. $250-287$. 
Sfelid, S., Jenssen, N. A: and Balchen, J. G. (1983). Design and analysis of a dynamic positioning system based on Kalman filtering and optimal control. IEEE Transactions on Automatic Contról, AC-28, pp. 331-339.

WiCHERS, J. E. W. (1993). Position control—from anchoring to DP system. New techniques for assessing and quantifying vessel stability and seakeeping qualities, University Enterprise Training Partnership. UETP, Marine Science and Technology Section 18. pp. 1-23 (Trondheim, Norway). 